\title{
Extremal edge polytopes
}

\author{
Tuan Tran* \\ Institute of Mathematics \\ Freie Universitt Berlin \\ Arnimallee 3, D-14195 Berlin, Germany \\ tuan@math.fu-berlin.de
}

\author{
Günter M. Ziegler** \\ Institute of Mathematics \\ Freie Universitt Berlin \\ Arnimallee 2, D-14195 Berlin, Germany \\ ziegler@math.fu-berlin.de
}

Submitted: Aug 5, 2013; Accepted: Jun 9, 2014; Published: Jun 27, 2014

Mathematics Subject Classifications: 05C35, 52B05, 52B12

\begin{abstract}
The edge polytope of a finite graph $G$ is the convex hull of the columns of its vertex-edge incidence matrix. We study extremal problems for this class of polytopes. For $k \in\{2,3,5\}$, we determine the maximal number of vertices of $k$ neighborly edge polytopes up to a sublinear term. We also construct a family of edge polytopes with exponentially-many facets.
\end{abstract}

Keywords: 0/1-polytopes; edge polytopes of graphs; subpolytopes of a hypersimplex; extremal $f$-vectors; number of facets; Turán numbers; pseudorandom graphs

\section{Introduction}

The main object of our investigation is a special class of 0/1-polytopes (cf. [31]): The edge polytope $\mathcal{P}(G)$ of a graph $G$ on the vertex set $\{1,2, \ldots, n\}$ is the polytope generated by all vectors $\mathbf{e}_{i}+\mathbf{e}_{j}$ such that $i$ is adjacent to $j$, where $\mathbf{e}_{i}$ and $\mathbf{e}_{j}$ stand for the $i$ th and $j$ th unit vectors of $\mathbb{R}^{n}$. For example, the edge polytopes of trees are simplices, while the edge polytope of the complete graph $K_{n}$ is the second hypersimplex $\Delta_{n-1}(2)$. Thus the edge polytopes are the subpolytopes of the second hypersimplex. A study of edge polytopes of general graphs was initiated by Ohsugi \& Hibi [25] and Villarreal [30], who both provided the half-space description of these polytopes (cf. Theorem 19). Dupont \& Villarreal [8] have recently connected this to the setting Rees Algebras in combinatorial commutative algebra. For further discussions of edge polytopes, see [12], [22], [24], and [26].

\footnotetext{
*Research supported by DFG within the Research Training Group "Methods for Discrete Structures".

** Research funded by the European Research Council under the European Union's Seventh Framework Programme (FP7/2007-2013) / ERC Grant agreement no. 247029-SDModels.
} 
In this paper we demonstrate that edge polytopes form a rich family of $0 / 1$-polytopes with interesting random and extremal properties. In particular, we obtain edge polytopes with an exponential number of facets (see Theorem 22) and $k$-neighborly 0/1-polytopes with more than linearly many vertices for any $k \geqslant 2$ (Corollary 17). On the other hand we will show that edge polytopes can be described and analyzed in terms of parameters of the graphs they are based on (and thus are not as intractable as the same problems for general 0/1-polytopes [16] [31]). Thus we obtain structural overview concerning three main topics:

(1) a description of the low-dimensional faces of the polytope $\mathcal{P}(G)$;

(2) non-linear relations between the components of the $f$-vector of $\mathcal{P}(G)$;

(3) the asymptotics of the maximal number of facets of $d$-dimensional edge polytopes for large $d$.

Here are some remarks connected to this. To describe all low-dimensional faces of $\mathcal{P}(G)$ we only need to consider "small" induced subgraphs of $G$. The second topic is closely related to the problem of finding minimal density of a fixed bipartite graph in a dense graph. Concerning the third topic Gatzouras et al. [10], improving on a breakthrough by Bárány

\& Pór [5], showed that there are random 0/1-polytopes in $\mathbb{R}^{d}$ with as many as $\left(\frac{c d}{\log ^{2} d}\right)^{d / 2}$ facets (or more), where $c>0$ is an absolute constant. The situation for $d$-dimensional random edge polytopes, however, where we have only a polynomial (quadratic) number of potential vertices, turns out to be quite different from that of general 0/1-polytopes.

The paper is divided into five sections. In the next section we introduce the object of our investigation and determine the dimension of an arbitrary edge polytope. A criterion for determining faces of edge polytopes is provided.

In Section 3 we compute the number of edges of $\mathcal{P}(G)$ in terms of the number of vertices of $\mathcal{P}(G)$, the number of 4-cycles and the number of 4-cliques in $G$. The function $g(n):=\max \left\{f_{1}(\mathcal{P}(G)): G\right.$ has $n$ vertices $\}$ is in Theorem 12 shown to be of order $\Theta\left(n^{4}\right)$. The lower bound for this is provided by random edge polytopes.

In Section 4 we characterize $k$-neighborly edge polytopes for $k \geqslant 2$. We then obtain a tight upper bound on the number of vertices of these edge polytopes, by counting various types of walks in the graph. All edge polytopes which attain these bounds are pseudo-random in some sense.

In Section 5 we use results of Ohsugi \& Hibi [25] to show that a $d$-dimensional edge polytope has at most $2^{d}+d$ facets. Inspired by Moon \& Moser [23], we provide a construction for $d$-dimensional edge polytopes with roughly $4^{d / 3}$ facets.

\section{Preliminaries}

All graphs in this paper are finite, undirected, with no loops, no multiple edges and no isolated vertices. The vertex and edge sets of a graph $G$ are denoted by $V(G)$ and $E(G)$. We write $|G|$ for the number of vertices of $G$, and $e(G)$ for the number of edges. We write $G[S]$ for the subgraph of $G$ induced by a set $S \subseteq V(G)$. Given two sets $S, T \subseteq V(G)$, not necessarily disjoint, we write $e_{G}(S, T)$ for the number of ordered pairs $(s, t)$ with 
$s \in S, t \in T$ and $s t \in E(G)$. Given a non-empty subset $X \subseteq V(G)$, the neighbor set of $X$ in $G$ is $N_{G}(X):=\{v \in V(G) \backslash X: v$ is adjacent to some vertex in $X\}$. If the graph $G$ is clear from the context, we often write $N(X)$ instead of $N_{G}(X)$.

The main object of our consideration is a special class of $0 / 1$-polytopes.

Definition 1. Let $G$ be a graph on the vertex set $[n]$. The edge polytope $\mathcal{P}(G)$ of $G$ is the convex hull of all vectors $\mathbf{e}_{i}+\mathbf{e}_{j}$ such that $i$ is adjacent to $j$, where $\mathbf{e}_{i}$ and $\mathbf{e}_{j}$ denote the $i$ th and $j$ th unit vectors of $\mathbb{R}^{n}$.

Thus the edge polytopes of $n$-vertex graphs correspond to the subpolytopes of the second hypersimplex of order $n$.

Example 2. The second hypersimplex of order $n$ is defined as

$$
\Delta_{n-1}(2):=\operatorname{conv}\left\{\mathbf{e}_{i}+\mathbf{e}_{j}: 1 \leqslant i<j \leqslant n\right\} \subseteq \mathbb{R}^{n} .
$$

It is the edge polytope of the complete graph $K_{n}$. The second hypersimplex $\Delta_{n-1}(2)$ has dimension $n-1$ (it is contained in $\left\{\mathbf{x} \in \mathbb{R}^{n}: x_{1}+\cdots+x_{n}=2\right\}$ ), $\left(\begin{array}{l}n \\ 2\end{array}\right)$ vertices, and $2 n$ facets if $n \geqslant 4$. For example, $\Delta_{3}(2) \subseteq \mathbb{R}^{4}$ is affinely equivalent to the regular octahedron.

Example 3. Let $C_{n}$ be the cycle $(1,2, \ldots, n-1, n)$. If $n$ is odd, then $\mathbf{e}_{1}+\mathbf{e}_{2}, \mathbf{e}_{2}+\mathbf{e}_{3}$, $\ldots, \mathbf{e}_{n-1}+\mathbf{e}_{n}, \mathbf{e}_{n}+\mathbf{e}_{1}$ are affinely independent, so the edge polytope $\mathcal{P}\left(C_{n}\right)$ is an $(n-1)$ simplex. If $n$ is even, then the edge polytope $\mathcal{P}\left(C_{n}\right)$ has dimension $n-2$ : It is a sum of two $\left(\frac{n}{2}-1\right)$-simplices in $\left\{\mathbf{x} \in \mathbb{R}^{n}: x_{1}+\cdots+x_{n}=2, \sum_{i=1}^{n}(-1)^{i} x_{i}=0\right\}$.

The definition of edge polytope can be rephrased in the following way. Let $G$ be a graph. The incidence matrix of $G$ is the matrix $A \in\{0,1\}^{V(G) \times E(G)}$ with

$$
a_{v, e}= \begin{cases}1 & \text { if } v \in e, \\ 0 & \text { otherwise }\end{cases}
$$

The edge polytope $\mathcal{P}(G)$ of $G$ is precisely the convex hull of the column vectors of the matrix $A$. Hence $\mathcal{P}(G)$ can be obtained by taking the intersection between the cone cone $(A)$ and the hyperplane $\left\{\mathbf{x} \in \mathbb{R}^{n}: x_{1}+\cdots+x_{n}=2\right\}$. Thus

$$
\operatorname{dim}(\mathcal{P}(G))=\operatorname{dim}(\operatorname{cone}(A))-1=\operatorname{rank}(A)-1 .
$$

On the other hand, by $\left[11\right.$, Theorem 8.2.1], $\operatorname{rank}(A)=n-c_{0}(G)$, where $c_{0}(G)$ is the number of connected bipartite components of $G$. We have therefore determined $\operatorname{dim}(\mathcal{P}(G))$.

Lemma 4 (Valencia \& Villarreal [29, p. 57]). Let $G$ be a graph with $n$ vertices and $c_{0}(G)$ bipartite components. Then the dimension of the edge polytope $\mathcal{P}(G)$ of $G$ equals $n-c_{0}(G)-1$.

This result enables us to obtain a quadratic upper bound on the number of vertices of the polytope $\mathcal{P}(G)$ in terms of its dimension. 
Proposition 5. Let $G$ be a finite graph, and let $d=\operatorname{dim}(\mathcal{P}(G))+1$. If $d \geqslant 4$, then $e(G) \leqslant\left(\begin{array}{l}d \\ 2\end{array}\right)$. Equality holds if and only if $G$ is a complete graph with at least 4 vertices.

For further investigation, we need a criterion for determining faces of an edge polytope. From now on, the symbol $\mathbf{e}_{i j}$ is used to denote the vector $\mathbf{e}_{i}+\mathbf{e}_{j}$.

We will use the following simple criterion.

Lemma 6. Let $V \subset \mathbb{R}^{n}$ be the vertex set of a polytope $P$ and let $U \subseteq V$. Then $U$ is the vertex set of a face of $P$ if and only if aff $(U) \cap \operatorname{conv}(V \backslash U)=\emptyset$.

For edge polytopes, this criterion can be reformulated as follows.

Lemma 7. Let $H$ be a subgraph of $G$ with the vertex set $U$. Then $\mathcal{P}(H)$ is a face of $\mathcal{P}(G)$ if and only if $\mathcal{P}(H)$ is a face of $\mathcal{P}(G[U])$.

Proof. The "only if" part follows from the fact that $\mathcal{P}(G[U])$ is a subpolytope of $\mathcal{P}(G)$ and $\mathcal{P}(H) \subseteq \mathcal{P}(G[U])$. For the "if" part, it is enough to show that $\mathcal{P}(G[U])$ is a face of $\mathcal{P}(G)$. Observe that all points $\mathbf{x} \in$ aff $\left\{\mathbf{e}_{i j}:\{i, j\} \in E(G[U])\right\}$ satisfy $\mathbf{x}_{i}=0$ for all $i \notin U$. Hence we have

$$
\text { aff }\left\{\mathbf{e}_{i j}:\{i, j\} \in E(G[U])\right\} \cap \operatorname{conv}\left\{\mathbf{e}_{i j}:\{i, j\} \in E(G) \backslash E(G[U])\right\}=\emptyset .
$$

So $\mathcal{P}(G[U])$ is a face of $\mathcal{P}(G)$, by Lemma 6 .

\section{Graphs of edge polytopes}

The following simple result of Ohsugi and Hibi identifies the edges of $\mathcal{P}(G)$.

Lemma 8 (Ohsugi \& Hibi [25]). The vertices $\mathbf{e}_{i j}$ and $\mathbf{e}_{k \ell}$ of $\mathcal{P}(G)$ form an edge if and only if

(i) the two edges $\{i, j\}$ and $\{k, \ell\}$ have a common vertex, or

(ii) $\{i, j\} \cap\{k, \ell\}=\emptyset,\{i, j\}$ and $\{k, \ell\}$ are not contained in any 4-cycle of $G$.

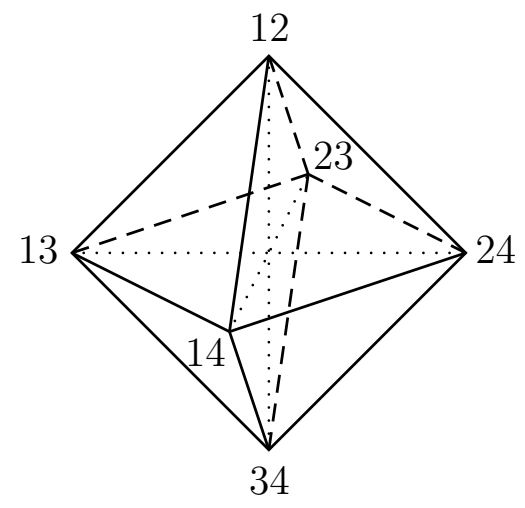

Figure 1: The octahedron $\mathcal{P}\left(K_{4}\right)$, a face of $\mathcal{P}\left(K_{n}\right)$. 
Using Lemma 8, we can compute the number of edges of $\mathcal{P}(G)$. For this, let $c_{4}(G)$ and $k_{4}(G)$ be the numbers of copies of $C_{4}$ and of $K_{4}$ in $G$, respectively. As usual in polytope theory, we denote by $f_{k}$ the number of $k$-dimensional faces of a polytope.

Proposition 9. If $\mathcal{P}(G)$ be the edge polytope of a simple graph $G$, then $f_{0}(\mathcal{P}(G))=e(G)$ and

$$
f_{1}(\mathcal{P}(G))=\left(\begin{array}{c}
e(G) \\
2
\end{array}\right)-2 c_{4}(G)+3 k_{4}(G) .
$$

Proof. Let $r_{4}(G)$ denote the number of pairs of disjoint edges of $G$ which are contained in some 4-cycle of $G$. Lemma 8 shows that $f_{1}=\left(\begin{array}{c}f_{0} \\ 2\end{array}\right)-r_{4}(G)$. Thus Proposition 9 will be established if $r_{4}(G)-2 c_{4}(G)+3 k_{4}(G)=0$ holds. Now

$$
r_{4}(G)-2 c_{4}(G)+3 k_{4}(G)=\sum_{U \subseteq[n],|U|=4}\left(r_{4}(G[U])-2 c_{4}(G[U])+3 k_{4}(G[U])\right),
$$

so we can assume from the beginning that $|G|=4$. The rest is left to the reader.

We next give a sharp lower bound for $f_{1}(\mathcal{P}(G))$ in terms of $f_{0}(\mathcal{P}(G))$.

Theorem 10. If $f_{0}$ and $f_{1}$ denote the number of vertices resp. edges of the edge polytope $\mathcal{P}(G)$, then

$$
f_{0}^{3 / 2}-f_{0} \leqslant f_{1}
$$

Equality holds if and only if $G$ is a complete bipartite graph with equal size parts.

Proof. Without restriction we can assume that $G$ is a connected graph on $n$ vertices. Let $\bar{d}$ be the average degree of $G$. Since $G$ is connected, it has at most one bipartite component. Thus we have $d:=\operatorname{dim}(\mathcal{P}(G))=n-c_{0}(G)-1 \geqslant n-2$. Now we count the following set $S$ in two ways: $S$ is the set of incidence pairs $(v, e)$ where $v$ is a vertex of $\mathcal{P}(G)$, and $e$ is an edge of $\mathcal{P}(G)$. Here $2 f_{1}=|S| \geqslant d f_{0}$, and hence $f_{1} \geqslant \frac{d f_{0}}{2}$. It follows that

$$
f_{1} \geqslant \frac{d f_{0}}{2} \geqslant \frac{(n-2) f_{0}}{2}=\frac{n f_{0}}{2}-f_{0} .
$$

Next we let $T$ be the set of pairs $(u,\{v, w\})$ where $u$ is adjacent to $v$ and to $w$, with $v \neq w$. Since $\{u, v\}$ and $\{u, w\}$ are two edges of $G$ which have one common node, they form an edge of $\mathcal{P}(G)$, by Lemma 8 . Therefore we have

$$
f_{1} \geqslant|T|=\sum_{v \in V(G)}\left(\begin{array}{c}
\operatorname{deg}(v) \\
2
\end{array}\right) \geqslant n\left(\begin{array}{c}
\bar{d} \\
2
\end{array}\right)=\frac{2 f_{0}^{2}}{n}-f_{0} .
$$

Combining this inequality with the previous one, we get

$$
f_{1} \geqslant \frac{1}{2}\left\{\frac{n f_{0}}{2}+\frac{2 f_{0}^{2}}{n}\right\}-f_{0} \geqslant f_{0}^{3 / 2}-f_{0} .
$$

It is easy to check that the equality holds if and only if $G \cong K_{n / 2, n / 2}$. 
Let $\mathcal{P}(G)$ be the edge polytope of a graph $G$ and $f_{1}(\mathcal{P}(G))$ be its number of edges. How large can $f_{1}(\mathcal{P}(G)$ ) be if the number of vertices of $G$ is fixed? Formally, we want to know the asymptotic behaviour of the number of edges of the polytope in terms of the number $n$ of vertices of the graph,

$$
g(n):=\max \left\{f_{1}(\mathcal{P}(G)):|G|=n\right\} .
$$

For that we use the following bound on the number of copies of a fixed complete bipartite subgraph of a graph of given density.

Lemma 11 (Alon [2, Corollary 2.1]). For every fixed $\varepsilon>0$, any two fixed integers $s \geqslant t \geqslant 1$, and for any graph $G$ with $n$ vertices and at least $\varepsilon n^{2}$ edges, the number of subgraphs of $G$ isomorphic to $K_{s, t}$ is at least

$$
(1+o(1))\left(\begin{array}{l}
n \\
s
\end{array}\right)\left(\begin{array}{l}
n \\
t
\end{array}\right)(2 \varepsilon)^{s t}
$$

if $s>t$, and at least

$$
\left(\frac{1}{2}+o(1)\right)\left(\begin{array}{l}
n \\
s
\end{array}\right)\left(\begin{array}{l}
n \\
t
\end{array}\right)(2 \varepsilon)^{s t}
$$

for $s=t$, where the $o(1)$ terms tend to 0 as $n$ tends to infinity.

It is worth noting that the assertions of the above lemma are tight, as shown by the random graph $G(n, 2 \varepsilon)$ on $n$ labeled vertices in which each pair of vertices is an edge with probability $2 \varepsilon$.

And now, as promised, we provide bounds for the function $g(n)$.

Theorem 12. For every integer $n \geqslant 6$, the function $g(n)=\max \left\{f_{1}(\mathcal{P}(G)):|G|=n\right\}$ satisfies

$$
\frac{1}{54} n^{4} \leqslant g(n) \leqslant\left(\frac{1}{32}+o(1)\right) n^{4} .
$$

Proof.

(i) Lower bound

For simplicity of notation, we write $\mathbb{G}$ instead of $G(n, p)$. Define

$$
p_{1}=\operatorname{Pr}\left(\mathbf{e}_{12}, \mathbf{e}_{13} \text { form an edge of } \mathcal{P}(\mathbb{G})\right)
$$

and

$$
p_{2}=\operatorname{Pr}\left(\mathbf{e}_{12}, \mathbf{e}_{34} \text { form an edge of } \mathcal{P}(\mathbb{G})\right) .
$$

Lemma 8 shows that conv $\left\{\mathbf{e}_{12}, \mathbf{e}_{13}\right\}$ is an edge of $\mathcal{P}(\mathbb{G})$ iff $\mathbf{e}_{12}, \mathbf{e}_{13} \in \mathbb{G}$, and thus $p_{1}=p^{2}$. To compute $p_{2}$, note that $\{1,2\}$ and $\{3,4\}$ are not contained in any 4-cycle of $\mathbb{G}$ if and only if not both $\{1,3\},\{2,4\}$ and not both $\{1,4\},\{2,3\}$ belong to $\mathbb{G}$ (see Figure 1 ). From this, we get $p_{2}=p^{2}\left(1-p^{2}\right)^{2}$. Thus by linearity of expectation

$$
\begin{aligned}
E f_{1} & =n\left(\begin{array}{c}
n-1 \\
2
\end{array}\right) p_{1}+\frac{1}{2}\left(\begin{array}{c}
n \\
2
\end{array}\right)\left(\begin{array}{c}
n-2 \\
2
\end{array}\right) p_{2} \\
& =n\left(\begin{array}{c}
n-1 \\
2
\end{array}\right) p^{2}+\frac{1}{2}\left(\begin{array}{c}
n \\
2
\end{array}\right)\left(\begin{array}{c}
n-2 \\
2
\end{array}\right) p^{2}\left(1-p^{2}\right)^{2} .
\end{aligned}
$$


For $p=1 / \sqrt{3}$ we get

$$
E f_{1}=n^{4} / 54+n^{3} / 18-8 n^{2} / 27+n / 3 \geqslant n^{4} / 54 .
$$

From this it follows that $g(n) \geqslant n^{4} / 54$.

(ii) Upper bound

Let $G$ be an arbitrary graph with $n$ vertices and $\rho \frac{n^{2}}{2}$ edges $(0<\rho<1)$. Since the cycle of length 4 is isomorphic to the complete bipartite graph $K_{2,2}$, Lemma 11 shows that $c_{4}(G) \geqslant\left(\frac{1}{8}+o(1)\right) \rho^{4} n^{4}$. Furthermore, as each clique of size 4 contains exactly three cycles of length 4 , we have $c_{4}(G) \geqslant 3 k_{4}(G)$. Therefore, the number of edges of $\mathcal{P}(G)$ is at most

$$
\begin{aligned}
\left(\begin{array}{c}
e(G) \\
2
\end{array}\right)-2 c_{4}(G)+3 k_{4}(G) & \leqslant\left(\begin{array}{c}
e(G) \\
2
\end{array}\right)-c_{4}(G) \\
& \leqslant \frac{1}{8} \rho^{2} n^{4}-\frac{1}{8} \rho^{4} n^{4}+o\left(n^{4}\right) \\
& \leqslant\left(\frac{1}{32}+o(1)\right) n^{4} .
\end{aligned}
$$

Remark. The upper bound in Theorem 12 is not tight: For any pair of graphs $F$ and $G$, let $N(F, G)$ denote the number of labeled copies of $F$ in $G$. A sequence $\left(G_{n}\right)$ of graphs $\left(\left|G_{n}\right| \rightarrow \infty\right)$ is quasi-random with density $p(0<p<1)$ if, for every graph $F$,

$$
N\left(F, G_{n}\right)=\left(p^{e(F)}+o(1)\right)\left|G_{n}\right|^{|F|} .
$$

According to Chung, Graham, and Wilson [7] this happens iff $N\left(K_{2}, G_{n}\right)=(p+o(1))\left|G_{n}\right|^{2}$ and $N\left(C_{4}, G_{n}\right)=\left(p^{4}+o(1)\right)\left|G_{n}\right|^{4}$.

If the upper bound of Theorem 12 is tight, then we can find a sequence $\left(G_{n}\right)$ of graphs such that $e\left(G_{n}\right)=\left(\frac{1}{\sqrt{8}}+o(1)\right)\left|G_{n}\right|^{2}, c_{4}\left(G_{n}\right)=\left(\frac{1}{32}+o(1)\right)\left|G_{n}\right|^{4}$, and $k_{4}\left(G_{n}\right)=\left(\frac{1}{96}+\right.$ $o(1))\left|G_{n}\right|^{4}$. This forces that $N\left(K_{2}, G_{n}\right)=\left(\frac{1}{\sqrt{2}}+o(1)\right)\left|G_{n}\right|^{2}, N\left(C_{4}, G_{n}\right)=\left(\frac{1}{4}+o(1)\right)\left|G_{n}\right|^{4}$, and $N\left(K_{4}, G_{n}\right)=\left(\frac{1}{4}+o(1)\right)\left|G_{n}\right|^{4}$. The first two equalities imply that $\left(G_{n}\right)$ is quasi-random with density $\frac{1}{\sqrt{2}}$. Hence $N\left(K_{4}, G_{n}\right)=\left(\frac{1}{8}+o(1)\right)\left|G_{n}\right|^{4}$, a contradiction.

It remains to be explored whether the upper bound can be improved by formalizing the subgraph count via flag algebras as in [14].

\section{Neighborly edge polytopes}

Here we provide a forbidden subgraph characterization for $k$-neighborly edge polytopes, and then determine the maximal number of vertices of such polytopes. For this we first prepare some notation.

Given a family $\mathcal{F}$ of graphs, a graph $G$ is $\mathcal{F}$-free if it contains no copy of a graph in $\mathcal{F}$ as a subgraph. The Turán number ex $(n, \mathcal{F})$ is the maximal number of edges in an $\mathcal{F}$-free graph on $n$ vertices. The Zarankiewicz number $\mathrm{z}(n, \mathcal{F})$ is the maximal number of edges in an $\mathcal{F}$-free bipartite graph on $n$ vertices.

A polytope $P$ is $k$-neighborly if every subset of at most $k$ of its vertices defines a face of $P$. Thus every polytope is 1-neighborly, and a polytope is 2-neighborly if and only 
if its graph is complete. Except for simplices, no $d$-dimensional polytope is more than $\left\lfloor\frac{d}{2}\right\rfloor$-neighborly.

For $k \geqslant 2$, let $\mathcal{F}_{k}$ be a family of graphs on at most $2 k$ vertices consisting of

- even cycles,

- graphs obtained by joining two odd cycles by a path.

For example, $\mathcal{F}_{2}=\left\{C_{4}\right\}$ and $\mathcal{F}_{3}=\left\{C_{4}, F_{2}, 2 K_{3}+e, C_{6}\right\}$ (see Figure 2).

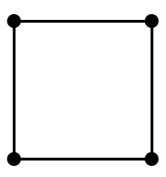

$C_{4}$

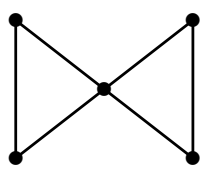

$F_{2}$



$2 K_{3}+e$

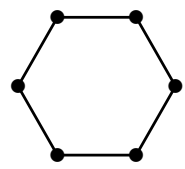

$C_{6}$

Figure 2: the family $\mathcal{F}_{3}$.

Now we can characterize $k$-neighborly edge polytopes for $k \geqslant 2$.

Theorem 13. For $k \geqslant 2$ the edge polytope $\mathcal{P}(G)$ of a graph $G$ with at least $k$ edges is $k$-neighborly if and only if $G$ is $\mathcal{F}_{k}$-free.

Before proving Theorem 13 we state a consequence. Let $\mathcal{C}_{2 k}^{\text {even }}$ denotes the family of all even cycles of lengths at most $2 k$. As a bipartite graph is $\mathcal{F}_{k}$-free if and only if it is $\mathcal{C}_{2 k}^{\text {even }}$-free, Theorem 13 implies the following result.

Corollary 14. For $k \geqslant 2$ the edge polytope $\mathcal{P}(G)$ of a bipartite graph $G$ with at least $k$ edges is $k$-neighborly if and only if $G$ is $\mathcal{C}_{2 k}^{\text {even }}$-free.

For the proof of Theorem 13, we use the following lemma, which is a straightforward consequence of results by Oshugi \& Hibi [25, Lemmas 1.4 and 1.5].

Lemma 15. Let $H$ be a finite graph. Then $\mathcal{P}(H)$ is a simplex if and only if every cycle in $H$ is odd, and every connected component of $H$ has at most one odd cycle.

This lemma can be reformulated in terms of forbidden subgraphs as follows.

Lemma 16. Suppose that $k \geqslant 2$ and $H$ is a graph with at most $2 k$ vertices. Then $H$ is $\mathcal{F}_{k}$-free if and only if every cycle in $H$ is odd, and every connected component of $H$ has at most one odd cycle.

Proof. The "if" part is obvious. For the "only if" part we observe that if a graph contains two odd cycles that intersect in more than one vertex, then it also has an even cycle.

We are now ready to prove Theorem 13 .

Proof of Theorem 13. Assume that $G$ is $\mathcal{F}_{k}$-free. Let $\left\{i_{1}, j_{1}\right\}, \ldots,\left\{i_{k}, j_{k}\right\}$ be $k$ different edges of $G$. Set $U=\left\{i_{1}, j_{1}, \ldots, i_{k}, j_{k}\right\}$, then $|U| \leqslant 2 k$. By Lemma 16 and Lemma 15, 
$\mathcal{P}(G[U])$ is a simplex. Therefore, $\operatorname{conv}\left\{\mathbf{e}_{i_{1} j_{1}}, \ldots, \mathbf{e}_{i_{k} j_{k}}\right\}$ is a face of $\mathcal{P}(G)$ by Lemma 7 . From this it follows that $\mathcal{P}(G)$ is $k$-neighborly.

Assume that $\mathcal{P}(G)$ is $k$-neighborly. Let $U \in\left(\begin{array}{c}V(G) \\ 2 k\end{array}\right)$ be arbitrary. If we set $\ell=$ $\min \{k, e(G[U])\}$, then the number of non-isolated vertices of $G[U]$ is at most $2 \ell$. Lemma 4 now shows that $\mathcal{P}(G[U])$ has dimension at most $2 \ell-1$. On the other hand, $\mathcal{P}(G[U])$ is $\ell$-neighborly by Lemma 7 . Hence $\mathcal{P}(G[U])$ is a simplex. Lemmas 15 and 16 imply that $G[U]$ is $\mathcal{F}_{k}$-free for each $U \in\left(\begin{array}{c}V(G) \\ 2 k\end{array}\right)$. Thus, $G$ is $\mathcal{F}_{k}$-free.

Theorem 13 can be used to obtain the following upper bound on the number of vertices of $k$-neighborly edge polytopes.

Corollary 17. Let $k \geqslant 2$ be a fixed integer. Then a $k$-neighborly edge polytope of an $n$-vertex graph has at most $\frac{1}{2} n^{1+1 / k}+\left(\frac{k-1}{2 k}+o(1)\right) n$ vertices.

Furthermore, for each $k \in\{2,3,5\}$ there are infinitely many positive integers $n$ for which there is an n-vertex graph $G_{n}$ whose edge polytope is $k$-neighborly with at least $\frac{1}{2} n^{1+1 / k}+\frac{k-1}{2 k} n-n^{1-1 / k}$ vertices.

Proof. In the following we will use results on Turán numbers and on pseudorandomness, Theorems 25 and 26, which are presented in the appendix.

Since $\mathcal{P}(G)$ is $k$-neighborly, Theorem 13 implies that the graph $G$ is $\mathcal{F}_{k}$-free. Under this condition we will show that, as $n$ goes to infinity, $e(G) \leqslant \frac{1}{2} n^{1+1 / k}+\left(\frac{k-1}{2 k}+o(1)\right) n$. We may assume that $e(G) \geqslant \frac{1}{2} n^{1+1 / k}$. Now let $\mathcal{T}$ be the set of all odd cycles of length at most $k$ in $G$, and let $U$ be the set of all vertices in $G$ which is contained in some element of $\mathcal{T}$. Because $G$ is $\mathcal{F}_{k}$-free, elements in $\mathcal{T}$ are pairwise disjoint, and consequently $|\mathcal{T}| \leqslant n / 3$. By removing one edge from each element of $\mathcal{T}$ we get a $\left(\mathcal{C}_{2 k}^{\text {even }} \cup \mathcal{C}_{k}\right)$-free graph $H$ with $e(H) \geqslant \frac{1}{2} n^{1+1 / k}-\frac{1}{3} n$, where $\mathcal{C}_{k}$ denotes the family of all cycles of lengths at most $k$. Since $H$ is $\left(\mathcal{C}_{2 k}^{\text {even }} \cup \mathcal{C}_{k}\right)$-free, Theorem 25 tells us that $e(H) \leqslant \frac{1}{2} n^{1+1 / k}+\frac{k-1}{2 k} n+n^{1-1 / k}$. Therefore, $H$ has average degree $d_{H} \sim n^{1 / k}$. Theorem 26 can be applied showing that

$$
e_{H}(S, T)=n^{-1+1 / k}|S||T|+o\left(n^{1+1 / k}\right) \quad \text { for every } S, T \subseteq V(G) .
$$

Since $H$ is obtained from $G$ by deleting $o\left(n^{1+1 / k}\right)$ edges, a similar formula holds for $G$, namely

$$
e_{G}(S, T)=n^{-1+1 / k}|S||T|+o\left(n^{1+1 / k}\right) \quad \text { for every } S, T \subseteq V(G) .
$$

As $G$ is $\mathcal{F}_{k}$-free, the induced subgraph $G[U]$ is the disjoint union of elements in $\mathcal{T}$. Hence $e_{G}(U, U)=2|U| \geqslant 6|\mathcal{T}|$. On the other hand, $e_{G}(U, U)=n^{-1+1 / k}|U|^{2}+o\left(n^{1+1 / k}\right)$. It follows that $|U|=o(n)$, and so $|\mathcal{T}|=o(n)$. From Theorem 25 we obtain

$$
e(G) \leqslant \operatorname{ex}\left(n, \mathcal{C}_{2 k}^{\text {even }} \cup \mathcal{C}_{k}\right)+|\mathcal{T}| \leqslant \frac{1}{2} n^{1+1 / k}+\left(\frac{k-1}{2 k}+o(1)\right) n .
$$

According to Theorem 25, for infinitely many positive integers $n$ there is a $\left(\mathcal{C}_{2 k}^{\text {even }} \cup \mathcal{C}_{k}\right)$ free graph $G_{n}$ on $n$ vertices such that $e\left(G_{n}\right) \geqslant \frac{1}{2} n^{1+1 / k}+\frac{k-1}{2 k} n-n^{1-1 / k}$. By Theorem 13, the edge polytopes of these graphs have the desired property.

We also have the following lower bound on the maximal number of vertices of a $k$-neighborly edge polytope. 
Corollary 18. Let $k \geqslant 2$ be a fixed integer. Then the following holds.

(i) A k-neighborly edge polytope of an $n$-vertex graph has at most $\frac{1}{2} n^{1+1 / k}+O(n)$ vertices. Moreover, there are graphs $G_{n}$ on $n$ vertices such that $\mathcal{P}\left(G_{n}\right)$ is a k-neighborly polytope with $\Omega\left(n^{1+2 / 3 k}\right)$ vertices.

(ii) A k-neighborly edge polytope of an n-vertex bipartite graph has at most $\left(\frac{1}{2}\right)^{1+1 / k} n^{1+1 / k}$ $+O(n)$ vertices. Moreover, for $k \in\{2,3,5\}$ this bound is tight up to the linear term for infinitely many $n$.

Proof. (i) If the edge polytope $\mathcal{P}(G)$ is $k$-neighborly, then $G$ is $\mathcal{C}_{2 k}^{\text {even }}$-free by Theorem 13 . Thus $\mathcal{P}(G)$ has at most $\operatorname{ex}\left(n, \mathcal{C}_{2 k}^{\text {even }}\right) \leqslant \frac{1}{2} n^{1+1 / k}+O(n)$ vertices, according to Lam \& Verstraëte [20]. On the other hand, Sarnak et al. [19] showed that $\operatorname{ex}\left(n,\left\{C_{3}, C_{4}, \ldots, C_{2 k}\right\}\right)=$ $\Omega\left(n^{1+2 / 3 k}\right)$. Thus Theorem 13 completes the proof.

(ii) If the edge polytope $\mathcal{P}(G)$ of a bipartite graph $G$ is $k$-neighborly, then the graph is $\mathcal{C}_{2 k}^{\text {even }}$-free by Theorem 13. It follows from [13] that the polytope $\mathcal{P}(G)$ has at most $\mathrm{z}\left(n, \mathcal{C}_{2 k}^{\text {even }}\right) \leqslant\left(\frac{1}{2}\right)^{1+1 / k} n^{1+1 / k}+O(n)$ vertices.

For $k \in\{2,3,5\}$ the existence of generalized polygons (Erdős \& Rényi [9], Benson [4], and Singleton [28]) shows that $\mathrm{z}\left(n, \mathcal{C}_{2 k}^{\text {even }}\right) \geqslant\left(\frac{1}{2}\right)^{1+1 / k} n^{1+1 / k}+O(n)$ for infinitely many $n$. Thus Corollary 14 completes the proof.

\section{$5 \quad$ Edge polytopes with many facets}

In this section we study the maximal number of facets of a $d$-dimensional edge polytope. Here we only deal with edge polytopes of connected graphs; all results can easily be extended to the general case.

We use some terminology of Ohsugi \& Hibi [25], as follows. Let $G$ be a connected graph on the vertex set $\{1,2, \ldots, n\}:=[n]$. A vertex $i \in[n]$ is regular (resp. ordinary) in $G$ if $G[[n] \backslash i]$ has no bipartite components (resp. if $G[[n] \backslash\{i\}]$ is connected).

A subset $\emptyset \neq A \subseteq[n]$ is independent in $G$ if $N(\{i\}) \cap A=\emptyset$ for all $i \in A$. If $A$ is independent in $G$, then the bipartite graph induced by $A$ in $G$ is defined to be the graph having the vertex set $A \cup N(A)$ and consisting of all edges $\{i, j\}$ of $G$ with $i \in A$ and $j \in N(A)$. This graph will be denoted by $G[A, N(A)]$.

When $G$ is non-bipartite, we say that a subset $\emptyset \neq A \subseteq[n]$ is fundamental in $G$ if

(i) $A$ is independent in $G$ and $G[A, N(A)]$ is connected, and

(ii) $G[[n] \backslash(A \cup N(A))]$ has no bipartite components.

When $G$ is bipartite, a subset $\emptyset \neq A \subseteq[n]$ is acceptable in $G$ if

(i) $A$ is independent in $G$ and $G[A, N(A)]$ is connected, and

(ii) $G[[n] \backslash(A \cup N(A))]$ is a connected graph with at least one edge.

Let $A$ be an non-empty independent set of $G$. Denoted by $\mathcal{H}_{A}^{+}$the closed half-space

$$
\mathcal{H}_{A}^{+}=\left\{\mathbf{x} \in \mathbb{R}^{n}: \sum_{i \in N(A)} x_{i}-\sum_{j \in A} x_{j} \geqslant 0\right\},
$$


and by $\mathcal{H}_{A}$ the hyperplane

$$
\mathcal{H}_{A}=\left\{\mathbf{x} \in \mathbb{R}^{n}: \sum_{i \in N(A)} x_{i}-\sum_{j \in A} x_{j}=0\right\} .
$$

If $i \in[n]$, then we write $\mathcal{H}_{i}^{+}$for the closed half-space

$$
\mathcal{H}_{i}^{+}=\left\{\mathbf{x} \in \mathbb{R}^{n}: x_{i} \geqslant 0\right\},
$$

and $\mathcal{H}_{i}$ for the hyperplane

$$
\mathcal{H}_{i}=\left\{\mathbf{x} \in \mathbb{R}^{n}: x_{i}=0\right\} .
$$

Theorem 19 (Ohsugi \& Hibi [25, Theorem 1.7]).

(i) Let $G$ be a connected non-bipartite graph on the vertex set $[n]$. Let $\Psi$ denote the set of those hyperplanes $\mathcal{H}_{i}$ such that $i$ is regular in $G$ and of those hyperplanes $\mathcal{H}_{A}$ such that $A$ is fundamental in $G$. Then the set of facets of the edge polytope $\mathcal{P}(G)$ is $\{\mathcal{H} \cap \mathcal{P}(G): \mathcal{H} \in \Psi\}$.

(ii) Let $G$ be a connected bipartite graph on the vertex set $V(G)=[n]$, and let $V(G)=$ $V_{1} \cup V_{2}$ be the partition of $V(G)$. Let $\Psi$ denote the set of those hyperplanes $\mathcal{H}_{i}$ such that $i$ is ordinary in $G$ and of those hyperplanes $\mathcal{H}_{A}$ such that $A$ is acceptable in $G$ with $A \subset V_{1}$. Then the set of facets of the edge polytope $\mathcal{P}(G)$ is $\{\mathcal{H} \cap \mathcal{P}(G): \mathcal{H} \in \Psi\}$.

Let $d \in \mathbb{N}$. We write $f(d)$ for the maximal number of facets of $\mathcal{P}(G)$, where $G$ ranges over all connected graph such that $\operatorname{dim}(\mathcal{P}(G))=d$.

Lemma 20. $f(d) \leqslant 2^{d}+d$ for all $d \geqslant 3$.

Proof. Let $G$ be a connected graph on $[n]$ with $\operatorname{dim}(\mathcal{P}(G))=d \geqslant 3$. Denote by $f_{d-1}$ the number of facets of $\mathcal{P}(G)$. It is sufficient to prove that $f_{d-1} \leqslant 2^{d}+d$. We distinguish two cases.

If $G$ is bipartite, then $d=n-2$. Let $V_{1} \cup V_{2}$ be the partition of $V(G)$. We can assume that $\left|V_{1}\right| \leqslant\left|V_{2}\right|$. Applying Theorem 19 , we get $f_{d-1} \leqslant 2^{\left|V_{1}\right|}+n \leqslant 2^{\lfloor n / 2\rfloor}+n \leqslant$ $2^{\lfloor(d+2) / 2\rfloor}+d+2<2^{d}+d$.

If $G$ is non-bipartite, then $d=n-1$. We denote by $\mathcal{F}$ the family of independent sets in $G$. Let $A \subseteq[n]$. Since $G$ is not bipartite, either $A \notin \mathcal{F}$ or $A^{c} \notin \mathcal{F}$. It follows that $|\mathcal{F}| \leqslant 2^{n-1}-1$. By Theorem 19, we see that $f_{d-1} \leqslant|\mathcal{F}|+n \leqslant\left(2^{n-1}-1\right)+n=2^{d}+d$.

Lemma 21. $f(d)>4^{\lfloor d / 3\rfloor}$ for all $d>0$.

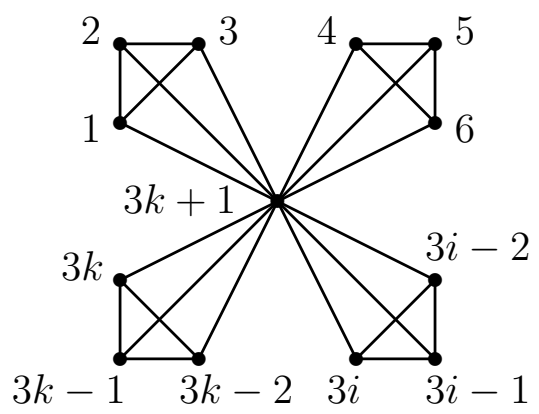

Figure 3: The windmill graph Wd $(4, k)$. 
Proof. Without restriction we can assume that $d=3 k$. Let $G$ be the windmill graph $\mathrm{Wd}(4, k)$ on the vertex set $[3 k+1]$ with the edge set

$$
\begin{aligned}
E(G)= & \{\{j, 3 k+1\}: j=1, \ldots, 3 k\} \cup \\
& \{\{3 i-2,3 i-1\},\{3 i-2,3 i\},\{3 i-1,3 i\}: i=1, \ldots, k\} .
\end{aligned}
$$

As $G$ is a connected non-bipartite graph, we have $\operatorname{dim}(\mathcal{P}(G))=(3 k+1)-1=d$. We will now determine all fundamental sets in $G$. Observe that a non-empty subset $A \subseteq[3 k+1]$ is independent in $G$ if and only if

(i) $A=\{3 k+1\}$, or

(ii) $3 k+1 \notin A$, and $|A \cap\{3 i-2,3 i-1,3 i\}| \leqslant 1$ for all $i=1, \ldots, k$.

We claim that such a set $A$ is fundamental in $G$. There are two possible cases. If $A=$ $\{3 k+1\}$, then $G[A, N(A)]$ is the graph with vertex set $[3 k+1]$ and edge set $\{\{j, 3 k+1\}$ : $j=1, \ldots, 3 k\}$. Since $A \cup N(A)=[3 k+1]$ and $G[A, N(A)]$ is connected, we see that $A$ is fundamental in $G$. If $3 k+1 \notin A$ and $|A \cap\{3 i-2,3 i-1,3 i\}| \leqslant 1$ for $i=\overline{1, k}$, then we can assume that $A=\{3,6, \ldots, 3 \ell\}$ for some $\ell \leqslant k$. In this case, we have

$$
\begin{gathered}
G[A, N(A)]=\{\{3 s, 3 s-2\},\{3 s, 3 s-1\},\{3 s, 3 k+1\}: s=1, \ldots, \ell\}, \\
G[\overline{A \cup N(A)}]=\{\{3 t-2,3 t-1\},\{3 t-2,3 t\},\{3 t-1,3 t\}: t=\ell+1, \ldots, k\} .
\end{gathered}
$$

Since $G[A, N(A)]$ is connected and $G[\overline{A \cup N(A)}]$ has no bipartite components, $A$ is fundamental in $G$.

We next claim that $i \in[3 k+1]$ is regular in $G$ if and only if $i \neq 3 k+1$. Indeed, we distinguish two cases. If $i=3 k+1$, then the induced subgraph $G[[3 k+1] \backslash i]$ is a disjoint union of $k$ triangles. It follows that $G[[3 k+1] \backslash i]$ is not connected, and consequently $i$ is not regular in $G$.

If $i \neq 3 k+1$, then we can assume that $i=1$. In this case, $G[[3 k+1] \backslash i]$ is the graph with vertex set $\{2,3, \ldots, 3 k+1\}$ and edge set

$$
\begin{aligned}
E= & \{\{j, 3 k+1\}: j=4, \ldots, 3 k\} \cup \\
& \{\{2,3\},\{2,3 k+1\},\{3,3 k+1\}\} \cup \\
& \{\{3 i-2,3 i-1\},\{3 i-2,3 i\},\{3 i-1,3 i\}: i=2, \ldots, k\} .
\end{aligned}
$$

We can see that this graph is connected and non-bipartite. Hence $i$ is a regular vertex in $G$, as desired. Finally, applying Theorem 19 we conclude that the number of facets of $\mathcal{P}(G)$ is $\sum_{\ell=1}^{k}\left(\begin{array}{l}k \\ \ell\end{array}\right) 3^{\ell}+3 k=4^{k}+3 k-1$. Therefore, $f(d)>4^{k}=4^{\lfloor/ 3\rfloor}$.

As a consequence of these lemmas, we obtain the following bounds on $f(d)$.

Theorem 22. For all $d \geqslant 3$, the maximal number $f(d)$ of facets of a d-dimensional edge polytope satisfies

$$
4^{\lfloor d / 3\rfloor}<f(d) \leqslant 2^{d}+d \text {. }
$$




\section{Appendix: Turán numbers}

Here we give a tight asymptotic upper bound on the Turán number $\operatorname{ex}\left(n, \mathcal{C}_{2 k}^{\text {even }} \cup \mathcal{C}_{k}\right)$, where $\mathcal{C}_{2 k}^{\text {even }}=\left\{C_{4}, C_{6}, \ldots, C_{2 k}\right\}$ and $\mathcal{C}_{k}=\left\{C_{3}, C_{4}, \ldots, C_{k}\right\}$. We also show that every nearly extremal $\left(\mathcal{C}_{2 k}^{\text {even }} \cup \mathcal{C}_{k}\right)$-free graph is pseudorandom.

The basic estimates for Turán numbers for even cycles are obtained by counting various types of walks in graphs: A non-returning walk of length $k$ in $G$ is a sequence $v_{0} e_{0} v_{1} e_{1} \ldots v_{k-1} e_{k-1} v_{k}$ such that $v_{i} \in V(G), e_{i}=\left\{v_{i}, v_{i+1}\right\} \in E(G)$, and $e_{i} \neq e_{i+1}$ for $0 \leqslant i<k$. Let $\nu_{k}(G)$ denote the average number of non-returning walks of length $k$ in $G$. If $G$ is a $d$-regular graph on $n$ vertices then clearly $\nu_{k}(G)=d(d-1)^{k-1}$. For irregular graphs, we have the following lower bound.

Proposition 23 (Alon, Hoory \& Linial [3]). If $G$ is a graph with minimum degree at least 2 and average degree $d$, then $\nu_{k}(G) \geqslant d(d-1)^{k-1}$.

The following simple result will be very useful for our investigation. It is probably well-known, but we couldn't find a reference for it.

Lemma 24. Suppose that $P$ and $Q$ are two different paths of length $k \geqslant 2$ with the same endpoints. If $P \cup Q$ is $\mathcal{C}_{k}$-free, then $P=\alpha P^{\prime} \beta$ and $Q=\alpha Q^{\prime} \beta$ for some vertex-disjoint paths $\alpha, \beta, P^{\prime}$ and $Q^{\prime}$.

Proof. The lemma is obviously true for $k \in\{2,3\}$. So let $k>3$ and proceed by induction. Let $x$ and $y$ be endpoints of $P$ and $Q$. We distinguish three cases.

Case 1: $N_{P}(y)=N_{Q}(y):=v$.

Let $P_{1}$ and $Q_{1}$ be the subpaths of $P$ and $Q$ from $x$ to $v$, respectively. Then they are two different paths of length $k-1$ from $x$ to $v$, and their union is $\mathcal{C}_{k}$-free. By induction, $P_{1}=\alpha P^{\prime} \beta$ and $Q_{1}=\alpha Q^{\prime} \beta$ for some vertex-disjoint paths $\alpha, \beta, P^{\prime}, Q^{\prime}$ with $x \in \alpha$ and $v \in \beta$. Hence $P=\alpha P^{\prime} \beta y$ and $Q=\alpha Q^{\prime} \beta y$.

Case 2: $N_{P}(y) \neq N_{Q}(y)$ and $N_{P}(y) \in Q$.

In this case, $N_{P}(y) Q y$ is a cycle of length at most $k$. This contradicts the assumption that $P \cup Q$ is $\mathcal{C}_{k}$-free.

Case 3: $N_{P}(x), N_{P}(y) \notin Q$ and $N_{Q}(x), N_{Q}(y) \notin P$.

We identify $x, N_{P}(x)$ and $N_{Q}(y)$ (resp. $y, N_{P}(y)$ and $N_{Q}(y)$ ) as a new vertex $x^{\prime}$ (resp. $y^{\prime}$ ). Let $P_{2}$ and $Q_{2}$ be the new paths corresponding to $P$ and $Q$. Then they are two different paths of length $k-2$ from $x^{\prime}$ to $y^{\prime}$. Since $P \cup Q$ is $\mathcal{C}_{k}$-free, $P_{2} \cup Q_{2}$ is $\mathcal{C}_{k-2}$-free. By induction $P_{2}=\alpha^{\prime} P^{\prime \prime} \beta^{\prime}$ and $Q_{2}=\alpha^{\prime} Q^{\prime \prime} \beta^{\prime}$ for some disjoint paths $\alpha^{\prime}, \beta^{\prime}, P^{\prime \prime}$ and $Q^{\prime \prime}$. We claim that $x^{\prime}$ is the only vertex of $\alpha^{\prime}$. Otherwise, $\alpha^{\prime}=x^{\prime} u \alpha^{\prime \prime}$ for some vertex $u$ and path $\alpha^{\prime \prime}$. In this case, the vertices $x, N_{P}(x), u$ and $N_{Q}(x)$ form a cycle of length 4 in $P \cup Q$, which contradicts the fact that $P \cup Q$ is $\mathcal{C}_{k}$-free. Similarly, $y^{\prime}$ is the only vertex of $\beta^{\prime}$. From this it follow that $P$ and $Q$ are two internally vertex-disjoint paths. Consequently, they have the desired structures.

Using the previous lemmas we can determine the Turán number of the family $\mathcal{C}_{2 k}^{\text {even }} \cup \mathcal{C}_{k}$ up to a sublinear term. 
Theorem 25. Suppose $k \geqslant 2$ and $n \geqslant 1$. Then

$$
\operatorname{ex}\left(n, \mathcal{C}_{2 k}^{\text {even }} \cup \mathcal{C}_{k}\right) \leqslant \frac{1}{2} n^{1+1 / k}+\frac{k-1}{2 k} n+n^{1-1 / k} .
$$

Furthermore, if $k \in\{2,3,5\}$, then for infinitely many $n$

$$
\operatorname{ex}\left(n, \mathcal{C}_{2 k}^{\text {even }} \cup \mathcal{C}_{k}\right) \geqslant \frac{1}{2} n^{1+1 / k}+\frac{k-1}{2 k} n-n^{1-1 / k} .
$$

It is quite well known (Kövári et al. [17] and Reiman [27], cf. [1, Chap. 25]) that $\operatorname{ex}\left(n, C_{4}\right) \leqslant\left\lfloor\frac{n}{4}(1+\sqrt{4 n-3})\right\rfloor$. On the other hand, Erdős \& Rényi [9] and Brown [6] proved that for infinitely many positive integer $n$ there is a $C_{4}$-free graph on $n$ vertices with $\frac{n-1}{4}(1+\sqrt{4 n-3})$ edges. These results establish Theorem 25 in the case when $k=2$. It remains to handle the case when $k \geqslant 3$.

Proof of Theorem 25. As discussed above, it is enough to prove the theorem for $k \geqslant 3$. Let $G$ be a minimal counterexample to the theorem. Then $G$ has minimum degree at least 2 and average degree $d>n^{1 / k}+\frac{k-1}{k}+2 n^{-1 / k}$. We denote by $\mathcal{P}_{k}$ the family of paths of length $k$ in $G$. Since $G$ is $\mathcal{C}_{k}$-free, every non-returning walk of length $k$ is nothing but a path of length $k$. It now follows from Proposition 23 that $\left|\mathcal{P}_{k}\right| \geqslant n d(d-1)^{k-1}>n^{2}$. By Lemma 24, for any ordered pair of vertices $u, v$ there is at most one path of length $k$ from $u$ to $v$. Thus, the number of paths of length $k$ is at most $n^{2}$, a contradiction.

Let $\alpha \in \mathbb{N}$. Set $q=2^{2 \alpha+1}$ if $k=3$, and $q=3^{2 \alpha+1}$ if $k=5$. Lazebnik et al. [21] constructed a $\left(\mathcal{C}_{2 k}^{\text {even }} \cup \mathcal{C}_{k}\right)$-free graph $G$ on $n=q^{k}+\ldots+q+1$ vertices with $\frac{1}{2}\left\{(q+1)\left(q^{k}+\right.\right.$ $\left.\ldots+q+1)-\left(q^{\left\lfloor\frac{k+1}{2}\right\rfloor}+1\right)\right\}$ edges. We can verify that $e(G) \geqslant \frac{1}{2} n^{1+1 / k}+\frac{k-1}{2 k} n-n^{1-1 / k}$ for large $n$.

Another key ingredient in the proof of Corollary 17 is the notion of pseudorandomness. We refer the reader to Krivelevich \& Sudakov [18] for a survey. The following result expresses the pseudorandomness property of a nearly extremal $\left(\mathcal{C}_{2 k}^{\text {even }} \cup \mathcal{C}_{k}\right)$-free graph: For any two large sets the number of ordered edges between them is close to what one would expect in a random graph of the same edge density.

Theorem 26. Let $k \geqslant 2$ be a fixed integer. Suppose $G$ is a $\left(\mathcal{C}_{2 k}^{\text {even }} \cup \mathcal{C}_{k}\right)$-free graph on $n$ vertices with average degree $d \sim n^{1 / k}$. Then $e_{G}(S, T)=\frac{d}{n}|S||T|+o\left(n^{1+1 / k}\right)$ for any $S, T \subseteq V(G)$.

Sketch. Our proof follows the lines of a remark of Keevash et al. [15, Section 9]. We just sketch the argument, and refer to [15] for the omitted details. Suppose that $G$ has eigenvalues $\lambda_{1} \geqslant \lambda_{2} \geqslant \cdots \geqslant \lambda_{n}$. Let $w_{2 k+2}^{\circ}(G)$ denote the number of closed walks of length $2 k+2$ in $G$ divided by $n$. Since $G$ is $\left(\mathcal{C}_{2 k}^{\text {even }} \cup \mathcal{C}_{k}\right)$-free, Lemma 24 shows that there is at most one path of length $k$ between any pair of vertices of $G$. Using this property we control the maximum degree as $\Delta<(1+\varepsilon) d$ by deleting $o\left(n^{1+1 / k}\right)$ edges. Then the argument of $[15$, Lemma 3.4] shows $w_{2 k+2}^{\circ}(G)<(1+o(1)) n \Delta^{2}$; the only difference is that there are $n-1$ choices for $u$ rather than $n / 2+o(n)$. On the other hand, $w_{2 k+2}^{\circ}(G)=\frac{1}{n} \sum \lambda_{i}^{2 k+2}$ has a contribution of $d^{2 k+2} / n \sim n d^{2}$ from the first eigenvalue, so the other eigenvalues are $o(d)$ as $\varepsilon \rightarrow 0$. The pseudorandomness property now follows from the non-bipartite version of [15, Lemma 5.4], which is provided in [18, Section 2.4]. 


\section{Acknowledgements.}

We are grateful to Raman Sanyal for helpful discussions, and Tibor Szabó for drawing our attention to the paper by Keevash, Sudakov \& Verstraëte [15]. We would like to thank an anonymous referee for very instructive comments and suggestions.

\section{References}

[1] Martin Aigner and Günter M. Ziegler. Proofs from THE BOOK. Fourth edition, Springer, Berlin, Heidelberg, 2010.

[2] Noga Alon. Testing subgraphs in large graphs. Random Structures and Algorithms, 21:359-370, 2002.

[3] Noga Alon, Shlomo Hoory, and Nati Linial. The Moore bound for irregular graphs. Graphs and Combinatorics, 18:53-57, 2002.

[4] Clark T. Benson. Minimal regular graphs of girths eight and twelve. Canadian Journal of Mathematics, 18:1091-1094, 1966.

[5] Imre Bárány and Attila Pór. On 0-1 polytopes with many facets. Advances in Mathematics, 161:209-228, 2001.

[6] William G. Brown. On graphs that do not contain a Thomsen graph. Canadian Mathematical Bulletin, 9:281-285, 1966.

[7] Fan Chung, Ronald L. Graham, and Richard M. Wilson. Quasi-random graphs. Combinatorica, 9:345-362, 1989.

[8] Louis A. Dupont and Rafael H. Villarreal. Symbolic Rees algebras, vertex covers and irreducible representations of Rees cones. Algebra and Discrete Mathematics, 10:64-86, 2010.

[9] Paul Erdős and Alfred Rényi. On a problem in the theory of graphs. Publications of the Mathematical Institute of the Hungarian Academy of Sciences, Ser. A, 7:623-641, 1962.

[10] Dimitris Gatzouras, Apostolos Giannopoulos, and Nikolaos Markoulakis. Lower bound for the maximal number of facets of a $0 / 1$ polytope. Discrete $\&$ Computational Geometry, 34:331-349, 2005.

[11] Chris Godsil and Gordon Royle. Algebraic Graph Theory. Graduate Texts in Mathematics, Vol. 207, Springer-Verlag, New York, 2001.

[12] Takayuki Hibi, Nan Li, and Yan X. Zhang. Separating hyperplanes of edge polytopes. Journal of Combinatorial Theory, Ser. A, 120:218-231, 2013.

[13] Shlomo Hoory. The size of bipartite graphs with a given girth. Journal of Combinatorial Theory, Ser. B, 86:215-220, 2002.

[14] Hao Huang, Nati Linial, Humberto Naves, Yuval Peled, and Benny Sudakov. On the 3-local profiles of graphs. Journal of Graph Theory, 76:236-248, 2014. 
[15] Peter Keevash, Benny Sudakov, and Jacques Verstraëte. On a conjecture of Erdős and Simonovits: Even cycles. Combinatorica, 33:699-732, 2013.

[16] Ulrich H. Kortenkamp, Jürgen Richter-Gebert, Aravamuthan Sarangarajan, and Günter M. Ziegler. Extremal properties of 0/1-polytopes. Discrete \& Computational Geometry, 17:439-448, 1997.

[17] Thomas Kővári, Vera T. Sós, and Paul Turán. On a problem of K. Zarankiewicz. Colloquium Mathematicum, 3:50-57, 1959.

[18] Michael Krivelevich and Benny Sudakov. Pseudo-random graphs. In More Sets, Graphs and Numbers, Bolyai Society Mathematical Studies, Vol. 15, 199-262, 2006.

[19] Alexander Lubotzky, Ralph Phillips, and Peter Sarnak. Ramanujan graphs. Combinatorica, 8:261-277, 1988.

[20] Thomas Lam and Jacques Verstraëte. A note on graphs without short even cycles. The Electronic Journal of Combinatorics, N5, 2005.

[21] Felix Lazebnik, Vasiliy A. Ustimenko, and Andrew J. Woldar. Polarities and 2kcycle-free graphs. Discrete Mathematics, 197/198:503-513, 1999.

[22] Tetsushi Matsui, Akihiro Higashitani, Yuuki Nagazawa, Hidefumi Ohsugi, and Takayuki Hibi. Roots of Ehrhart polynomials arising from graphs. Journal of Algebraic Combinatorics, 34:721-749, 2011.

[23] John W. Moon and Leo Moser. On cliques in graphs. Israel Journal of Mathematics, 3:23-28, 1965.

[24] Hidefumi Ohsugi and Takayuki Hibi. Compressed polytopes, initial ideals and complete multipartite graphs. Illinois Journal of Mathematics, 44:391-406, 2000.

[25] Hidefumi Ohsugi and Takayuki Hibi. Normal polytopes arising from finite graphs. Journal of Algebra, 207:409-426, 1998.

[26] Hidefumi Ohsugi and Takayuki Hibi. Simple polytopes arising from finite graphs. In Proc. 2008 Int. Conf. Information Theory and Statistical Learning (ITSL 2008), Las Vegas, July 14-17, 2008 (M. Dehmer et al., eds.), CSREA Press 2008, 73-79.

[27] Istvan Reiman. Über ein Problem von K. Zarankiewicz. Acta Mathematica Academiae Scientiarum Hungaricae, 9:269-278, 1958.

[28] Robert Singleton. On minimal graphs of maximum even girth. Journal of Combinatorial Theory, 1:306-332, 1966.

[29] Carlos E. Valencia and Rafael H. Villarreal. Explicit representations of the edge cone of a graph. International Journal of Contemporary Mathematical Sciences, 1:53-66, 2006.

[30] Rafael H. Villarreal. On the equations of the edge cone of a graph and some applications. Manuscripta Mathematica, 97:309-317, 1998.

[31] Günter M. Ziegler. Lectures on 0/1-polytopes. In Polytopes-Combinatorics and Computation (G. Kalai \& G. M. Ziegler, eds.), DMV Seminars, Vol. 29, Birkhäuser, Basel 2000, pages 1-41. 